\title{
One-dimensional Josephson arrays as superlattices for single Cooper pairs
}

\author{
A. A. Odintsov \\ Department of Applied Physics, Delft University of Technology, 2628 CJ Delft, The Netherlands \\ and Nuclear Physics Institute, Moscow State University, Moscow 119899 GSP, Russia \\ (Received 10 October 1995; revised manuscript received 15 March 1996)
}

\begin{abstract}
We investigate uniform one-dimensional arrays of small Josephson junctions $\left[E_{J} \ll E_{C}, E_{C}=(2 e)^{2} / 2 C\right]$ with a realistic Coulomb interaction $U(x)=E_{C} \lambda \exp (-|x| / \lambda)$ (here $\lambda \gg 1$ is the screening length in units of the lattice constant of the array). At low energies this system can be described in terms of interacting Bose particles (extra single Cooper pairs) on the lattice. With increasing concentration $\nu$ of extra Cooper pairs, a crossover from the Bose gas phase to the Wigner crystal phase and then to the superlattice regime occurs. The phase diagram in the superlattice regime consists of commensurable insulating phases with $\nu=1 / l$ ( $l$ is integer) separated by superconducting regions where the current is carried by excitations with fractional electric charge $q= \pm 2 e / l$. The Josephson current through a ring-shaped array pierced by magnetic flux is calculated for all of the phases. [S0163-1829(96)08026-5]
\end{abstract}

\section{INTRODUCTION}

The investigation of arrays of small Josephson junctions has attracted growing interest by theoreticians and experimentalists (see Ref. 1 for a review). In such arrays the Coulomb energy $E_{C}=(2 e)^{2} / 2 C$ can be of the order of the Josephson energy $E_{J}$ (here $C$ is the capacitance of the junctions). Since the Josephson phase $\phi$ and the electric charge $Q$ on the islands are canonically conjugated operators, $[\phi, Q]=2 e i$, a number of nontrivial quantum phenomena arise due to a competition between the phase (or vortex) and charge degrees of freedom. In particular, the point of the superconductor-insulator transition depends on the magnetic frustration $^{2}$ and on the electrochemical potential $\mu$ of the array. $^{3-6}$

Existing theories of electric-field-induced superconductorinsulator transition ${ }^{3-6}$ treat predominantly the cases of onsite or nearest-neighbor Coulomb interactions, although for typical experimental parameters the range of the interaction is large, ${ }^{1} \lambda=3-20$ (in units of the lattice constant of the array). The phase diagram of the superconductor-insulator transition becomes rather complicated for $\lambda \gg 1$ and large concentrations $\nu \sim 1 / \lambda^{d}$ of electric-field-induced ("extra") Cooper pairs ${ }^{5}$ (here $d$ is the dimension of the array). This is related to the fact that the extra Cooper pairs (ECP's) can form a variety of configurations commensurable with the lattice of junctions. On the other hand, it is known ${ }^{7}$ that at very small concentrations ${ }^{8} \nu \ll 1 / \lambda^{d}$ the ECP's form a Bose gas with a hard-core interaction and commensurability with the lattice plays no role. In this work we study the transition between these two very different regimes.

We consider uniform one-dimensional (1D) Josephson arrays with large Coulomb energy $E_{C} \gg E_{J}$ and long-range Coulomb interactions, $\lambda \gg 1$. We focus on the regime with low concentrations $\nu$ of ECP's,

$$
\exp (-1 / \lambda \nu) \ll 1
$$

In this regime the problem can be reduced to the effective Hamiltonian of impenetrable bosons on the lattice (Sec. II).
The estimates of parameters (Sec. III) show that at low concentrations the ECP's form incommensurable phases: Bose gas and Wigner crystal. With increasing concentration the system enters the superlattice regime where commensurability with the lattice becomes important. We analyze the phase diagram of the superconductor-insulator transition in this regime in Sec. IV. In the insulating phase the ECP's form a regular superlattice with the period $l(\nu=1 / l)$. The system starts showing superconducting properties when the first mobile excitation appears. The latter corresponds to a change of the distance between two neighboring ECP's in a superlattice by $\Delta l= \pm 1$. This excitation can be treated as a quasiparticle with fractional charge $q=\mp 2 e / l$. The superconducting phase can be viewed as a gas of such quasiparticles on the lattice.

Our estimates show that the superconductor-insulator transition can be observed experimentally by measuring the Josephson current through a circular array pierced by a magnetic flux. This current is evaluated for all the phases. Finally, we discuss some specific effects due to a finite size of an array. The conclusions are presented in Sec. V.

\section{EFFECTIVE HAMILTONIAN}

The Hamiltonian $H=H_{C}+H_{J}$ of a 1D Josephson array consists of a Coulomb term $H_{C}$ and a Josephson term $H_{J}$. The Coulomb energy is given by

$$
H_{C}=\frac{1}{2} \sum_{i, j=1}^{L} n_{i} U(i-j) n_{j}-\mu N
$$

where $n_{i}$ is a (positive or negative) number of ECP on the electrode $i, \quad N=\sum_{i=1}^{L} n_{i}$, and the interaction $U(i-j)=2 e^{2}\left(C^{-1}\right)_{i, j}$ is determined by the inverse of the capacitance matrix $C_{i, j}$. A standard model ${ }^{1}$ of the Josephson array accounts for the capacitance $C$ of the Josephson junctions and the capacitance $C_{g}$ of the superconducting islands to the ground. In this case the interaction potential can be approximated by 


$$
U(x)=E_{C} \lambda \exp (-|x| / \lambda),
$$

where $\lambda=\left(C / C_{g}\right)^{1 / 2}$. The gate voltage $V$ plays the role of the chemical potential, $\mu=2 \mathrm{eV}$.

We consider first the limit of zero Josephson coupling. For $|\mu|<\mu_{\mathrm{tr}} \equiv \lambda E_{C} / 2$ there is no ECP's in the array $\left(n_{i}=0\right.$ for all $i$ ). Just above the threshold, $0<\mu-\mu_{\mathrm{tr}} \ll \mu_{\mathrm{tr}}$, the ground state is still characterized by the absence of ECP's on most of the islands $\left(n_{i}=0\right)$. The rest of the islands are occupied by one ECP $\left(n_{i}=1\right)$. The configurations with $n_{i}$ $\neq 0,1$ (for some $i$ ) are separated from the ground state by a Coulomb gap $\Delta_{C}=E_{C} / \lambda$, which corresponds to the difference between the Coulomb energies of the configurations $\left\{n_{i}\right\}=(0, \ldots, 1,-1,1,0, \ldots)$ and $(0, \ldots, 1,0, \ldots)$. In what follows we restrict the space of states to low-energy configurations with $n_{i}=0$ or 1 . These configurations can be fully characterized by the coordinates $x_{j}$ of ECP's on the lattice $\left(x_{j}\right.$ are integer numbers).

The Josephson term of the Hamiltonian has a standard form

$$
H_{J}=-E_{J} \sum_{i=1}^{L} \cos \left(\phi_{i+1}-\phi_{i}-a\right)
$$

Here $\phi_{i}$ is the operator of the Josephson phase of the island $i$ obeying the commutation relation $\left[n_{i}, e^{ \pm i \phi_{k}}\right]$ $= \pm \delta_{i, k} e^{ \pm i \phi_{k}}$, and $a=(2 \pi / L) \Phi / \Phi_{0}$ is the vector potential (for circular array pierced by a magnetic flux $\Phi$ ). The Josephson term acting on the restricted space of states describes a hoping of ECP's on the lattice with the amplitude $E_{J} e^{ \pm i a} / 2$. Corrections to the tunneling amplitude due to the states with $n \neq 0,1$ are small for

$$
E_{J} \ll \Delta_{C} .
$$

Therefore, the original Hamiltonian $H$ in the low-energy space is equivalent to the Hamiltonian of Bose particles (ECP's) on the lattice, ${ }^{9}$

$$
H=-E_{J} \sum_{j=1}^{N} \cos \left(p_{j}-a\right)+\sum_{j=1}^{N} U\left(x_{j}-x_{j-1}\right)-\bar{\mu} N
$$

where $p_{j}$ are quasimomenta of ECP's and $\bar{\mu}=\mu-\mu_{\mathrm{tr}}$. The pairs $\left(x_{j}, p_{j}\right)$ are conjugate action-angle operators satisfying $\left[e^{ \pm i p_{j}}, x_{k}\right]= \pm \delta_{j, k} e^{ \pm i p_{j}}$. We have taken into account only the interaction of the neighboring ECP's in Eq. (6), which is legitimate in the regime (1) under consideration.

\section{ESTIMATES OF PARAMETERS}

Let us consider now the ground state of the system as a function of the chemical potential $\bar{\mu}$ at fixed $E_{J}$. The first ECP enters the array at $\bar{\mu}=-E_{J}$. We denote the deviation from this threshold by $\tilde{\mu}=\bar{\mu}+E_{J}$. At very low concentrations of ECP's (a precise condition will be given below) the effect of interaction is negligible, unless two particles come close (to distances $\sim \lambda$ ) to each other. Therefore, to a good approximation, ECP's form a Bose gas with a hard-core interaction. ${ }^{7}$ To treat this regime we use the trial ground-state wave function

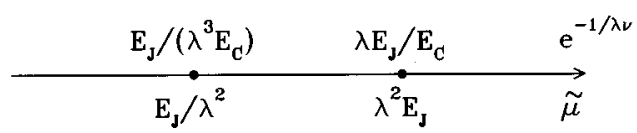

$\begin{array}{llcc}\text { State: } & \text { Bose gas } & \text { Wigner crystal } & \text { Superlattice } \\ \text { Interaction: } & \text { unharmonic } & \text { harmonic } & \text { harmonic } \\ \text { Band: } & \text { parabolic } & \text { parabolic } & \cos p\end{array}$

FIG. 1. Schematic phase diagram of a 1D Josephson array.

$$
\Psi=\prod_{i<j} \sin \frac{\pi\left|x_{i}-x_{j}\right|}{L} \Theta\left(\left|x_{i}-x_{j}\right|-d\right),
$$

where the size $d$ of the core plays a role a variational parameter. The kinetic energy per particle in the state (7) is given by ${ }^{10}$

$$
K_{1 p}=\frac{\pi^{2} E_{J} \nu^{2}}{6(1-\nu d)^{2}} .
$$

Since $K_{1 p} \ll E_{J}$, the energy band is effectively parabolic, $-E_{J} \cos \left(p_{j}-a\right) \rightarrow E_{J}\left(p_{j}-a\right)^{2} / 2-E_{J}$ in Eq. (6). The potential energy per particle can be evaluated as

$$
U_{1 p}=\frac{2 \pi^{2}}{3} \frac{(\nu \lambda)^{3}}{(1-\nu d)^{2}} E_{C} \lambda e^{-d / \lambda}
$$

for $\nu d \ll 1$. Minimizing the total energy we obtain the optimum value for $d$,

$$
d_{0}=\lambda \ln \left(\frac{2 E_{C} \lambda^{3}}{E_{J}}\right)
$$

The chemical potential is given by

$$
\widetilde{\mu}^{(\mathrm{BG})}=\frac{\pi^{2} E_{J} \nu^{2}}{2}\left[1+O\left(\nu d_{0}\right)\right] .
$$

This expression coincides with the chemical potential of a 1D gas of impenetrable bosons up to the term $O\left(\nu d_{0}\right)$. A variational ansatz (7) breaks down when $d_{0}$ becomes comparable to the interparticle distance $1 / \nu$, i.e., when

$$
\exp \left(-\frac{1}{\nu \lambda}\right) \sim \frac{E_{J}}{\lambda^{3} E_{C}}
$$

This condition determines the upper boundary of the range of concentrations in which a hard-core approximation of the interaction is applicable (Fig. 1).

With increasing concentration the ECP's get localized in coordinate space. We assume a priori that the ECP's form a 1D Wigner crystal. We introduce the deviations $u_{j}=x_{j}-x_{j-1}-l$ of the distance between neighboring particles from its average value $l=\nu^{-1}$ and expand the potential energy $U(x)$, Eq. (6), up to quadratic in $u_{j}$ terms. Expanding also the kinetic energy we obtain a quadratic Hamiltonian, which enables one to estimate the characteristics of the system. In particular, the kinetic energy per particle $K_{1 p}$ and the fluctuation $\left\langle u_{j}^{2}\right\rangle$ of the interparticle distance are given by

$$
K_{1 p}=\pi^{-1} \sqrt{2 E_{J} U_{0}},\left\langle u_{j}^{2}\right\rangle=\pi^{-1} \sqrt{2 E_{J} / U_{0}},
$$


where $U_{0} \equiv(1 / 2) d^{2} U(x) /\left.d x^{2}\right|_{x=l}$ is a stiffness of the Wigner lattice,

$$
U_{0}(l)=\left(E_{C} / 2 \lambda\right) \exp (-l / \lambda) .
$$

The chemical potential is given by

$$
\tilde{\mu}^{(\mathrm{wC})}=\lambda E_{C} e^{-1 / \lambda \nu}+\frac{1}{\pi \nu} \sqrt{\frac{E_{C} E_{J}}{\lambda^{3}}} e^{-1 / 2 \lambda \nu},
$$

where the first term is related to the energy of a static deformation of the lattice of ECP's, and the second term corresponds to the energy of the normal modes of the lattice (phonons). Note that the concentration $\nu$ of ECP's increases as a function of the chemical potential $\tilde{\mu}$ much more slowly than in the Bose gas regime.

The expansion of the potential and kinetic energy up to quadratic terms is legitimate if the fluctuations of $u_{i}$ are small, $\left\langle u_{i}^{2}\right\rangle \ll \lambda^{2}$, and the kinetic energy per ECP's is much less than $E_{J}$. These conditions determine respectively the lower and the upper bounds of the range of concentrations,

$$
\frac{E_{J}}{\lambda^{3} E_{C}} \ll \exp \left(-\frac{1}{\nu \lambda}\right) \ll \frac{\lambda E_{J}}{E_{C}},
$$

in which the Wigner crystal phase exists (Fig. 1). Note that the lower estimate (16) for the Wigner crystal is consistent with the (upper) estimate (12) for the Bose gas.

For both the phases (Bose gas and Wigner crystal) the Hamiltonian is quadratic in momenta of the particles. Therefore, the vector potential $a$ is coupled to the momenta of the center of mass only. For this reason the Josephson current through a ring-shaped array is given by a universal expression

$$
I_{J}=\frac{4 e v_{J}}{L} \frac{\Phi}{\Phi_{0}}, \quad v_{J}=\frac{\pi \nu E_{J}}{\hbar},
$$

for $|\Phi|<\Phi_{0} / 2$. A similar result holds for the persistent current of interacting fermions, ${ }^{11} v_{J}$ being the Fermi velocity in that case. Equation (17) enables one to estimate the scale of the Josephson current through a circular array. For a choice of parameters $L=100, \nu=0.1$, and $E_{J}=1 \mathrm{meV}$ we obtain $I_{J} \simeq 1.6 \mathrm{nA}$ (for $\Phi=\Phi_{0} / 2$ ). It seems that this current can be measured using modern experimental technique. ${ }^{12}$

For larger concentrations of ECP's

$$
\frac{\lambda E_{J}}{E_{C}} \ll \exp \left(-\frac{1}{\nu \lambda}\right),
$$

the kinetic energy per particle becomes comparable to the bandwidth $E_{J}$ and the effects of the lattice become important (see Fig. 1).

\section{SUPERLATTICE REGIME}

To investigate this regime we start from the limit $E_{J} \rightarrow 0$. In this case the ground state will correspond to configurations of ECP's commensurable with the array of Josephson junctions. First, we determine the range of the chemical potential,

$$
\mu_{l,-}(0)<\bar{\mu}<\mu_{l,+}(0),
$$

where the simplest commensurable configuration with equidistantly spaced (at a distance $l$ ) ECP's is the ground state of the system. One can say that all neighboring ECP's are connected by "bonds" of length $l$ in this configuration. In order to add (subtract) a Cooper pair into (from) this configuration, one has to convert $l-1(l+1)$ bonds of length $l$ into $l$ bonds of length $l-1(l+1)$. The energy required for this conversion determines the chemical potentials,

$$
\mu_{l, \pm}(0)= \pm[l U(l \mp 1)-(l \mp 1) U(l)] \simeq \mu_{l} \pm \epsilon_{l} / 2 \lambda,
$$

with $\mu_{l}=\epsilon_{l}(1+\lambda / l), \quad \epsilon_{l}=E_{C} l e^{-l / \lambda}$. One sees that $\mu_{l,+}(0)=\mu_{l-1,-}(0)$. Therefore, the ground state corresponds to a regular superlattice of ECP's in the array; the period of the superlattice changes abruptly from $l$ to $l-1$ at $\bar{\mu}=\mu_{l,+}(0)$; see Fig. 2(a). This simple picture of the ground state is valid if one takes into account the interaction of neighboring ECP's only [see Eq. (6)]. The interaction of next-nearest neighbors will lead to new ground states ${ }^{13}$ in exponentially narrow regions $\left|\bar{\mu}-\mu_{l,+}(0)\right| \sim \epsilon_{l} e^{-l / \lambda}$ near the points $\mu_{l,+}(0)$.

\section{A. Superconductor-insulator transition}

First, we consider a stability of commensurable phase (a superlattice of ECP's with period $l$ ) in the presence of a small but finite Josephson coupling, $E_{J} \ll U_{0}(l)$. The commensurable phase can be destroyed by mobile excitations (or quantum defects) which can be viewed as bonds of the length $l \pm 1$ surrounded by regular bonds of length $l$. Let us consider a bond of length $l+1$. The tunneling of ECP's on the left (right) end of the bond to one lattice cell to the right (left) will bring the excitation to $l$ cells left (right). This means that the excitation has fractional charge ${ }^{14}-2 e / l$. Analogously, the excitation of the length $l-1$ has the charge $2 e / l$.

Tunneling of mobile excitations decreases the energy of the system by an amount $E_{J}$ per excitation [see the first term of Eq. (6)]. This makes it more energetically favorable for mobile excitations to enter the system, i.e., shrinks the range (19) of the commensurable phase. The boundary of commensurable phase can be determined by equating the total energies of the system with and without a mobile excitation [this is the case for any ratio between $E_{J}$ and $\left.U_{0}(l)\right]$. Since addition (subtraction) of one Cooper pair to (from) the array is accompanied by the creation of $l$ mobile excitations, the threshold chemical potentials (20) for the commensurable phase are modified as follows,

$$
\mu_{l, \pm}\left(E_{J}\right)=\mu_{l, \pm}(0) \mp l E_{J} .
$$

We now turn to the evaluation of the boundary between the commensurable (insulating) and incommensurable (superconducting) phases for an arbitrary $E_{J}$. In the superlattice regime (18) under consideration the fluctuations of the interparticle distances $u_{j}=x_{j}-x_{j-1}-l$ are small, $\left\langle u_{j}^{2}\right\rangle \ll \lambda^{2}$ [cf. Eq. (16)] and we can again expand the potential energy $U(x)$, Eq. (6), up to quadratic in $u_{j}$ terms (see Fig. 1). Let us recall that the operators $u_{j}$ and $x_{j}$ have integer eigenvalues since they describe the positions of the particles on the lattice. We introduce an operator $\varphi_{j}$ canonically conjugated to 

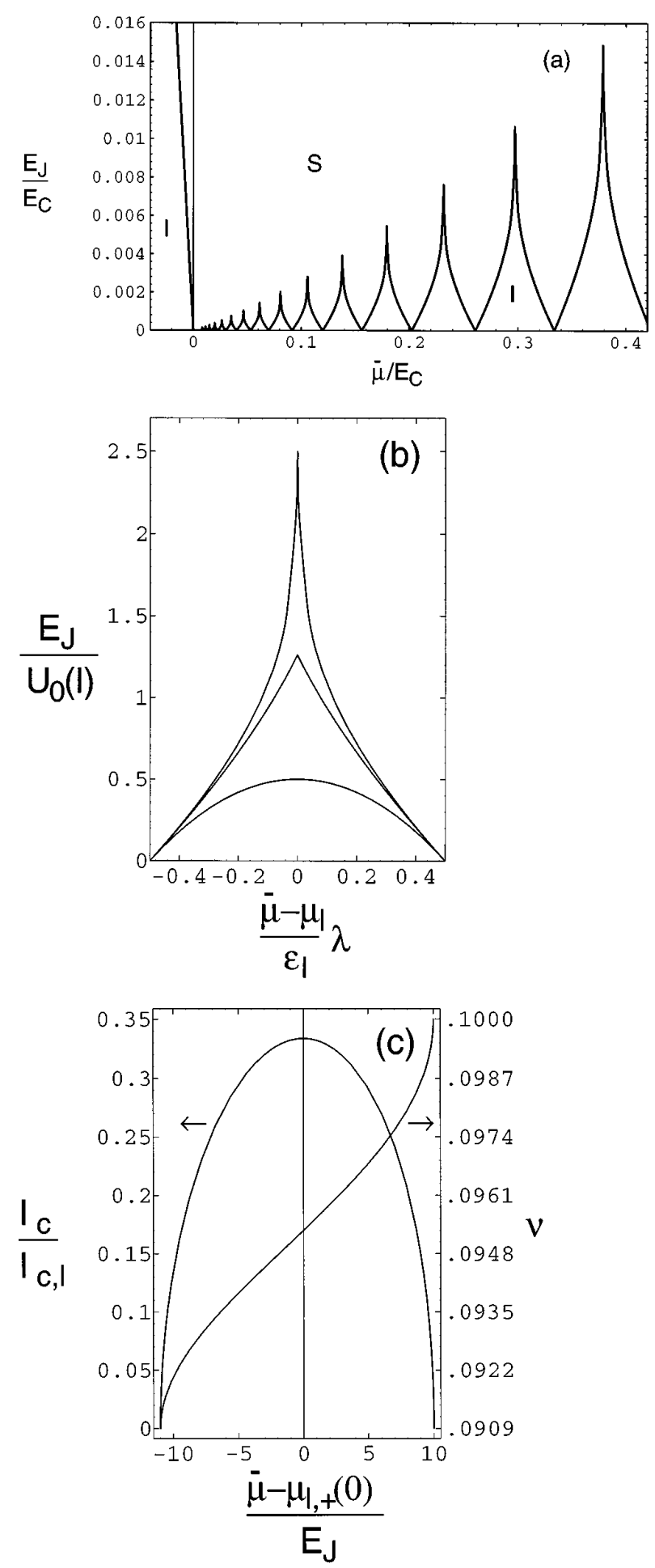

FIG. 2. Phase diagram of the superconductor-insulator transition in a 1D Josephson array $(\lambda=3)$. (a) The overall view. The insulating spikelike regions from right to left correspond to $\nu=1 / 10,1 / 11, \ldots$. (b) Boundary of the insulating phase with $\nu=1 / l$. The curves from bottom to top correspond to the results of the mean-field approach, the third-order perturbation theory, and the extrapolation of the perturbation theory to infinite order connected with the extrapolation of the Kosterlitz-Thouless scaling. (c) Concentration $\nu(\bar{\mu})$ of ECP's and critical Josephson current $I_{c}(\bar{\mu})$ in the superconducting region $1 / 11<\nu<1 / 10(l=11)$. We normalize $I_{c}$ by the critical current (5) for the concentration $\nu=1 / l$ of ECP's, $I_{c, l}=2 \pi e E_{J} / \hbar l L$. $u_{j}$ which satisfies $\left[e^{ \pm i \varphi_{j}}, u_{k}\right]= \pm \delta_{j, k} e^{ \pm i \varphi_{j}}$. The operator $\varphi_{j}$ is related to the operators $p_{j}$ [see (6)] of quasimomenta of ECP's, $p_{j}=\varphi_{j}-\varphi_{j+1}$. The number of particles, $N$, can be expressed via $u_{j}, N=\left(L-\Sigma u_{j}\right) / l$. As a result, the Hamiltonian (6) can be written as

$$
H=-E_{J} \sum_{j} \cos \left(\varphi_{j+1}-\varphi_{j}+a\right)+U_{0}(l) \sum_{j}\left(u_{j}+\delta \mu\right)^{2},
$$

where $\delta \mu=\lambda\left(\bar{\mu}-\mu_{l}\right) / \epsilon_{l}$. Hence, we have shown that the Hamiltonian of a Josephson array with a long-range (but screened) Coulomb interaction in the superlattice regime can be reduced to the Hamiltonian (22) which formally describes a 1D Josephson array with an on-site interaction. This Hamiltonian has been extensively studied in Refs. $3-5,15$ in the context of Josephson arrays and Bose Hubbard model.

As we have already discussed, the boundary of the commensurable phase can be determined by equating the groundstate energies of the Hamiltonian (22) in two subspaces of states: with $\Sigma u_{j}=0$ (this corresponds to the absence of mobile excitations) and with $\Sigma u_{j}= \pm 1$ (i.e., in the presence of one mobile excitation). In the limit $E_{J} \rightarrow 0$ the ground states in these subspaces are given by $\Psi_{0}\left(u_{1}, \ldots, u_{N}\right)=\Pi_{j} \delta_{u_{j}, 0}$ and $\Psi_{ \pm 1}\left(u_{1}, \ldots, u_{N}\right)=N^{-1 / 2} \Sigma_{k} \delta_{u_{k}, \pm 1} \Pi_{j \neq k} \delta_{u_{j}, 0}$. Evaluating the energies of these states up to the third order in $E_{J}$ (see Ref. 4) we obtain

$$
\mu_{l, \pm}\left(E_{J}\right)=\mu_{l} \pm\left\{\frac{\epsilon_{l}}{2 \lambda}-l E_{J}\left[1-\frac{E_{J}}{8 U_{0}(l)}-\frac{E_{J}^{2}}{32 U_{0}^{2}(l)}\right]\right\},
$$

for $E_{J} \ll U_{0}(l)$; see Fig. 2(b). Note that up to a term linear in $E_{J}$ this equation coincides with the result (21) obtained from a simple argument.

With increasing $E_{J}$ the range of the chemical potential corresponding to commensurable phase shrinks and both phase boundaries tend to the critical point, $\mu_{l, \pm}\left(E_{J}^{(\mathrm{cr})}\right) \rightarrow \mu_{l}$; see Fig. 2(b). Clearly, the true behavior near the critical point cannot be described by perturbation theory of finite order. To extend the perturbative approach, an extrapolation to infinite order in $E_{J}$ was proposed. ${ }^{4} \mathrm{Un}$ fortunately, this (somewhat speculative) extrapolation fails to converge to a critical point for the 1D system.

To determine the behavior near the critical point $E_{J}^{(\mathrm{cr})}$ one can map the Hamiltonian (22) (with $\bar{\mu}=\mu_{l}$ ) onto 2-D $X Y$ model. ${ }^{15}$ The parameter $\left[2 U_{0}(l) / E_{J}\right]^{1 / 2}$ plays the role of a dimensionless temperature $k_{B} T / J$ in the $X Y$ model. The point of the Kosterlitz-Thouless transition ${ }^{16}$ corresponds to $E_{J}^{(\mathrm{cr})} \cong 2.5 U_{0}(l)$; see Fig. 2(b). Below the transition temperature $\left(E_{J}>E_{J}^{(\mathrm{cr})}\right)$ spin-spin correlations in the $X Y$ model decay algebraically with distance. The Josephson array shows superconducting properties: the Josephson current is inversely proportional to $L$. It scales as ${ }^{15} 1+c \sqrt{E_{J}-E_{J}^{(\mathrm{cr})}}$ at $E_{J} \rightarrow E_{J}^{(\mathrm{cr})}+0$ (here $c$ is a nonuniversal constant). Above the transition temperature $\left(E_{J}<E_{J}^{(\mathrm{cr})}\right)$ the correlations in the $X Y$ model decay exponentially. Near the critical point $\left(E_{J} \rightarrow E_{J}^{(\mathrm{cr})}-0\right)$ the coherence length is given by $\xi=\exp \left\{-b\left[E_{J}^{(\mathrm{cr})} /\left(E_{J}^{(\mathrm{cr})}-E_{J}\right)\right]^{1 / 2}\right\}$, where $b \simeq 2$. As a result, 
the Josephson current through a 1D array decays as $\exp (-L / l \xi)$, signaling the formation of the insulating phase.

In the insulating phase, the energy gap for the mobile excitations scales as ${ }^{3,4} \xi^{-1}$. For this reason, the boundary of the insulating phase near the critical point is given by

$$
\mu_{l, \pm}\left(E_{J}\right)=\mu_{l} \pm \alpha l U_{0}(l) / \xi
$$

with $\alpha \sim 1$. The upper curve in Fig. 2(b) corresponds to an extrapolation of Eq. (24) from the neighborhood of the critical point to lower values of $E_{J}\left[1.56 U_{0}(l)<E_{J}<E_{J}^{(\mathrm{cr})}\right]$. This extrapolation is joined to the extrapolation of the perturbative result (23) to infinite order in $E_{J}$ [drawn in the range $\left.E_{J}<1.56 U_{0}(l)\right]$. A smooth connection of the two curves occurs for $\alpha \simeq 1.73$ (and $b=2$ ).

For completeness, we present the mean-field result for the phase boundary, ${ }^{3,5}$

$$
\mu_{l, \pm}\left(E_{J}\right)=\mu_{l} \pm \frac{\epsilon_{l}}{2 \lambda} \sqrt{1-\frac{2 E_{J}}{U_{0}(l)}} .
$$

Although this expression coincides with Eq. (23) to first order in $E_{J}$, the overall shape of the boundary [Fig. 2(b)] and the critical value of the Josephson energy $E_{J}^{(\mathrm{cr})}$ differ considerably from the results discussed above. The reason for the failure of the mean-field approach is the absence of longrange order in one dimension.

\section{B. Superconducting phase}

We consider now the incommensurable superconducting phase and concentrate on the case of small Josephson coupling, $E_{J} \ll U_{0}(l)$. In this case the incommensurable phase exists in narrow regions $\mu_{l,+}\left(E_{J}\right)<\bar{\mu}<\mu_{l-1,-}\left(E_{J}\right)$ of the width $(2 l-1) E_{J}$ between two commensurable phases with $\nu=1 / l$ and $\nu=1 /(l-1)$ [Fig. 2(a)].

In this region the ground state can be well approximated by the configurations of ECP's containing bonds of length $l$ and $l-1$ only. These configurations form a reduced space of states. The other configurations can be ignored since the energy of the bonds of the length different from $l$ and $l-1$ is of the order of $U_{0}(l)$ which is much larger than average energy $\sim E_{J}$ per bond in the ground state.

The problem (6) in the reduced space is equivalent to impenetrable Bose particles (bonds of length $l-1$ ) on a lattice formed by all the bonds (equivalently, one can consider the bonds of length $l$ as particles). Note that there is no interaction between these particles which is a consequence of the fact that only the interaction between neighboring ECP's was taken into account in (6). The concentration $q=N_{1} / N$ of bonds of length $l-1$ can be expressed via the concentration $\nu=N / L$ of ECP's, $q=l-\nu^{-1} \quad[0<q<1$ in the range $1 / l<\nu<1 /(l-1)$ under consideration]. The energy of the system is then given by

$$
E=-E_{J} \cos a \frac{\sin (\pi q)}{\sin (\pi / N)}-\left[\bar{\mu}-\mu_{l,+}(0)\right] N,
$$

where the first term is the energy of $N_{1}$ impenetrable bosons on the lattice. Using Eq. (26) we obtain the chemical poten- tial $\bar{\mu}$ and the Josephson current $I_{J}$ through a circular array as functions of the concentration $\nu$ of ECP's,

$$
\begin{gathered}
\bar{\mu}=\mu_{l,+}(0)-E_{J}\left\{(l-q) \cos \pi q+\pi^{-1} \sin \pi q\right\}, \\
I_{J}=\frac{4 e E_{J}}{\hbar L} \frac{\sin \pi q}{l-q} \frac{\Phi}{\Phi_{0}},
\end{gathered}
$$

for $|\Phi|<\Phi_{0} / 2$. These dependences are presented in Fig. 2(c).

\section{Finite-size effects}

Finally, in view of possible experimental implementation, we discuss effects which are specific for finite-size circular arrays. If the size $L$ of the array is commensurable with the spacing $l$ of the superlattice of ECP's, the Josephson current is exponentially small in the insulating phase. However, if $L / l$ is not an integer, a number of residual mobile excitations remain in the array in the insulating phase, $\mu_{l,-}\left(E_{J}\right)<\bar{\mu}<\mu_{l,+}\left(E_{J}\right)$. In the lower part of this range, $\mu_{l,-}\left(E_{J}\right)<\bar{\mu}<\mu_{l, 0}\left(E_{J}\right)$, there are $m=\bmod (L, l)$ residual excitations (bonds of the length $l+1$ ) in the ground state. One ECP's enters into the array at $\bar{\mu}=\mu_{l, 0}\left(E_{J}\right)$. As a result, for $\mu_{l, 0}\left(E_{J}\right)<\bar{\mu}<\mu_{l,+}\left(E_{J}\right)$ a new ground state will contain $l-m$ residual excitations (bonds of the length $l-1$ ). The threshold chemical potential $\mu_{l, 0}\left(E_{J}\right)$ can be evaluated by comparing the energies of the residual excitations in both cases,

$$
\mu_{l, 0}\left(E_{J}\right)=\left[m \mu_{l,-}\left(E_{J}\right)+(l-m) \mu_{l,+}\left(E_{J}\right)\right] / l .
$$

Since each excitation contributes an amount $I_{1 C P}=\left(4 \pi e E_{J} / \hbar L^{2}\right)\left(\Phi / \Phi_{0}\right)$ to the Josephson current $[\mathrm{cf}$. Eq. (17) with $\nu=1 / L]$, the latter shows a jump $\Delta I=|l-2 m| I_{1 \mathrm{CP}}$ at $\bar{\mu}=\mu_{l, 0}\left(E_{J}\right)$.

\section{CONCLUSIONS}

In this paper we have investigated uniform 1D Josephson arrays in the charging regime $E_{C} \gg E_{J}$. We considered a realistic long-range (but screened) Coulomb interaction and concentrated on the case of low charge frustration which provides a low concentration of extra charges (Cooper pairs) in the array.

This regime can be described by an effective low energy Hamiltonian (6). We found a crossover from the Bose gas phase to the Wigner crystal phase with increasing concentration of extra Cooper pairs. At still higher concentrations the system enters the superlattice regime. In this regime a true phase transition between commensurable (insulating) and incommensurable (superconducting) phases occurs. We found a simple structure of the phase diagram. In the insulating phase the extra Cooper pairs form a regular superlattice with the period $l$, whereas the superconducting phase can be viewed as a gas of fractionally charged excitations on the lattice.

The boundary of the commensurable phase can be determined analitycally for small Josephson coupling $E_{J} \ll U_{0}(l)$ [using perturbation theory; see Eq. (23)] and in the vicinity of a critical point $E_{J}^{(\mathrm{cr})}$ [by mapping the problem onto clas- 
sical $X Y$ model; see Eq. (24)]. We have also computed the Josephson current through a circular array pierced by a magnetic flux (for all the phases) and considered the effects of a finite size of the array.

There is clearly a need for future investigations, such as an analysis of effects of disorder due to the offset charges (potential disorder) and due to nonuniformity of the Josephson coupling (kinetic disorder).

\section{ACKNOWLEDGMENTS}

I would like to thank Yu.V. Nazarov and A. Shelankov for useful discussions and P. Hadley for a critical reading of the manuscript. The financial support of the European Community through Grant No. ERB-CHBI-CT94-1474 is gratefully acknowledged. This work is also a part of INTAS-93790 project.
${ }^{1}$ Single Charge Tunneling, edited by H. Grabert and M. H. Devoret Vol. 294 of NATO Advanced Study Institute, Series B: Physics (Plenum, New York, 1992), Chaps. 7, 8.

${ }^{2}$ M. P. A. Fisher, Phys. Rev. Lett. 65, 923 (1990); H. S. J. van der Zant, F. C. Fritschy, W. J. Elion, L. J. Geerligs, and J. E. Mooij, ibid. 69, 2971 (1992).

${ }^{3}$ M. P. A. Fisher, P. B. Weichman, G. Grinstein, and D. S. Fisher, Phys. Rev. B 40, 546 (1989); E. Roddick and D. Stroud, ibid. 48, 16600 (1993).

${ }^{4}$ J. K. Freericks and H. Monien, Europhys. Lett. 26, 545 (1994); Phys. Rev. B 53, 2691 (1996).

${ }^{5}$ C. Bruder, R. Fazio, A. Kampf, A. van Otterlo, and G. Schön, Phys. Scr. 42, 159 (1992); C. Bruder, R. Fazio, and G. Schön, Phys. Rev. B 47, 342 (1993); A. van Otterlo, K. Wagenblast, R. Baltin, C. Bruder, R. Fazio, and G. Schön, Phys. Rev. B 52, 16176 (1995).

${ }^{6}$ G. G. Batrouni, R. T. Scalettar, G. T. Zimanyi, and A. P. Kampf, Phys. Rev. Lett. 74, 2527 (1995).

${ }^{7}$ A. A. Odintsov and Yu. V. Nazarov, Phys. Rev. B 51, 1133 (1995).
${ }^{8}$ For simplicity we omit logarithmic factor in this estimate; see Eq. (12).

${ }^{9}$ Formally, $H$ can be mapped also on the Hamiltonian of fermions on the lattice; see Ref. 10.

${ }^{10}$ E. H. Lieb and W. Liniger, Phys. Rev. 1301605 (1963); M. Girardeau, J. Math. Phys. 1, 516 (1960).

${ }^{11}$ F. D. M. Haldane, Phys. Rev. Lett. 47, 1840 (1981); D. Loss, ibid. 69, 343 (1992).

${ }^{12}$ D. Mailly, C. Chapelier, and A. Benoit, Phys. Rev. Lett. 70, 2020 (1993).

${ }^{13}$ J. Hubbard, Phys. Rev. B 17, 494 (1978).

${ }^{14}$ Fractionally charged excitations occur in 1D systems like charge density waves and polymer chains. These systems are more complicated than a Josephson array because of the presence of the electron and phonon subsystems; see, e.g., Electronic Properties of Quasi-One-Dimensional Compounds, edited by P. Monceau (Reidel, Dordrecht, 1985).

${ }^{15}$ R. M. Bradley and S. Doniach, Phys. Rev. B 30, 1138 (1984).

${ }^{16}$ J. M. Kosterlitz and D. J. Thouless, J. Phys. C 6, 1181 (1973); J. M. Kosterlitz, ibid. 17, 1046 (1974). 